\title{
Die Rechtsfigur des urheberrechtlichen Freihaltebedürfnisses zu Gunsten der Allgemeinheit
}

OGH, Urteil vom 14. Oktober 2008 - 4 Ob 162/08i

\begin{abstract}
1. Urheberrechtlich schützbar sind Werke nur, wenn sie eigentümlich iSd § 1 Abs. 1 ÖsterrUrhG sind. Diese Beschränkung folgt im Wesentlichen aus dem urheberrechtlichen Freihaltebedürfnis zu Gunsten der Allgemeinheit. Nur eine individuell eigenartige Leistung, die sich vom Alltäglichen, Landläufigen, üblicherweise Hervorgebrachten abhebt, ist geschützt.
\end{abstract}

2. Ein einzelner Werkteil ist nur geschützt, wenn er für sich eine eigentümliche geistige Schöpfung iSd § 1 Abs. 1 ÖsterrUrhG ist. Um den Schutz eines einzelnen Werkteils zu beurteilen, ist es somit weder erforderlich, diesen in eine Relation zum Gesamtwerk zu stellen, noch den Werkcharakter des Gesamtwerks zu beurteilen. (Leitsätze der Redaktion)

\section{Spruch}

- Dem Rekurs wird Folge gegeben.

Der angefochtene Beschluss wird aufgehoben und in der Sache selbst dahin zu Recht erkannt, dass das Ersturteil wie folgt zu lauten hat:

„Das Klagebegehren,

a) die Beklagte sei schuldig, es zu unterlassen, Skulpturen herzustellen, zu vervielfältigen oder zu vertreiben, die aus Schokolade bestehende Frauenschuhe darstellen, wie sie auf den beigeschlossenen, einen integrierenden Bestandteil des Urteils bildenden Fotos Beil./A dargestellt sind;

b) die Beklagte sei schuldig, der Klägerin für die Nutzung des Werks ein angemessenes Entgelt zuzüglich Umsatzsteuer zu bezahlen, wobei der Klägerin dessen ziffernmäßige Festsetzung bis zur Rechnungslegung vorbehalten bleibt;

c) die Beklagte sei schuldig, für die Nutzung des seit Dezember 2005 vervielfältigten und verbreiteten Werks Schadenersatz in Höhe des doppelten angemessenen Entgelts zu leisten, insoweit dieser das angemessene Entgelt übersteigt;

d) die Klägerin zu ermächtigen, den stattgebenden Teil des Urteils betreffend das Unterlassungsbegehren auf näher bestimmte Weise veröffentlichen zu lassen;

e) die Beklagte sei schuldig, sämtliche in ihrer Verfügung befindlichen Originale und Vervielfältigungen des Werks sowie die zur widerrechtlichen Vervielfältigung bestimmten Mittel nachweislich zu vernichten;

f) die Beklagte sei schuldig, der Klägerin über das widerrechtlich hergestellte Werk Rechnung zu legen und die Richtigkeit der gelegten Rechnung durch einen allgemein gerichtlich beeideten Sachverständigen auf Kosten der Beklagten prüfen zu lassen,
Die klagende Partei ist schuldig, der beklagten Partei die mit 5.581,92 EUR (darin 930,32 EUR USt) bestimmten Prozesskosten binnen 14 Tagen zu ersetzen."

Die klagende Partei ist schuldig, der beklagten Partei die mit 6.167,78 EUR (darin 677,63 EUR USt und 2.102 EUR Barauslagen) bestimmten Kosten des Rechtsmittelverfahrens binnen 14 Tagen zu ersetzen.

\section{Entscheidungsgründe}

Die Klägerin ist Projekt- und Performancekünstlerin. Sie hat an den Kunstakademien von Venedig und Wien studiert und ihre Werke in zahlreichen Ausstellungen vorgestellt. 1993 wurde sie eingeladen, an der Ausstellung des Rheinischen Landesmuseums Bonn mit dem Thema "Der Schuh als Kunstobjekt" teilzunehmen. Die Klägerin stellte dort einen Videofilm mit dem Titel

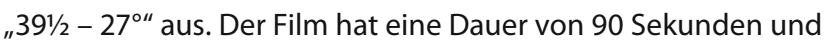
zeigt, wie ein Mann einer Frau einen Schokoladeschuh vom Fuß isst. Für die Aufnahme dieses Films ließ die Klägerin einen Schokoladeschuh in Konfektionsgröße herstellen, den man tragen, allerdings auch essen konnte. Seit dieser Ausstellung werden im Auftrag der Klägerin regelmäßig Reproduktionen dieses Schuhs in verschiedenen Geschmacksrichtungen (Vollmilch, Zartbitter, Erdbeer u.a.) durch Nachgießen erzeugt und in eigens dafür angefertigten Schachteln mit Satineinlage in Museumsläden zum Kauf angeboten [...].

2005 erteilte die Klägerin der Beklagten, einer weltbekannten Konditorei in Wien, den Auftrag, den Schuh aus Schokolade nachzugießen, und übergab ihr dazu eine Gussform, die nach Auftragserfüllung bei der Beklagten verblieb. Die Beklagte fertigte mit Hilfe dieser Gussform 2006 ohne Wissen und Einverständnis der Klägerin mehrere Schuhe aus Zuckerguss an, die sie zur Gestaltung ihrer Auslage verwendete [...]; zumindest einen aus Schokolade nachgegossenen Schuh hat die Beklagte auch verkauft.

Die Klägerin stellte folgende - ausschließlich auf urheberrechtliche Bestimmungen gestützte - Begehren:

wird abgewiesen. 
a) Die Beklagte ist schuldig, es zu unterlassen, Skulpturen herzustellen, zu vervielfältigen oder zu vertreiben, die aus Schokolade bestehende Frauenschuhe darstellen, wie sie auf den beigeschlossenen, einen integrierenden Bestandteil des Urteils bildenden Fotos Beil./A dargestellt sind;

b) die Beklagte ist schuldig, der Klägerin für die Nutzung des Werks ein angemessenes Entgelt zuzüglich Umsatzsteuer zu bezahlen, wobei der Klägerin dessen ziffernmäßige Festsetzung bis zur Rechnungslegung vorbehalten bleibt;

c) die Beklagte ist schuldig, für die Nutzung des seit Dezember 2005 vervielfältigten und verbreiteten Werks Schadenersatz in Höhe des doppelten angemessenen Entgelts zu leisten, insoweit dieser das angemessene Entgelt übersteigt;

d) die Klägerin wird ermächtigt, den stattgebenden Teil des Urteils betreffend das Unterlassungsbegehren auf näher bestimmte Weise veröffentlichen zu lassen;

e) die Beklagte ist schuldig, sämtliche in ihrer Verfügung befindlichen Originale und Vervielfältigungen des Werks sowie die zur widerrechtlichen Vervielfältigung bestimmten Mittel nachweislich zu vernichten;

f) die Beklagte ist schuldig, der Klägerin über das widerrechtlich hergestellte Werk Rechnung zu legen und die Richtigkeit der gelegten Rechnung durch einen allgemein gerichtlich beeideten Sachverständigen auf Kosten der Beklagten prüfen zu lassen.

Das von der Klägerin geschaffene Objekt sei Ergebnis einer schöpferischen geistigen Tätigkeit; es sei eigenartig, unterscheide sich von anderen Werken und reflektiere die Persönlichkeit seiner Schöpferin. Auch das Produzieren von gewöhnlichen Gegenständen aus einem ungewöhnlichen Stoff sei eine eigentümliche geistige Leistung, die urheberrechtlichen Schutz genieße. Die Klägerin habe mit ihrem aus Schokolade bestehenden Schuh ein Kunstwerk schaffen wollen, vergleichbar mit der Schokoladepuppe von Dieter Roth oder den "Readymades" von Marcel Duchamp. Dadurch, dass die Beklagte ohne Zustimmung der Klägerin aus den ihr zur Verfügung gestellten Formen weitere Skulpturen gegossen, diese in geringfügig veränderter Fassung und minderer Qualität in ihrer Auslage und im Lokal ausgestellt und sogar verkauft habe, habe sie Urheberrechte der Klägerin verletzt. Die Beklagte wendete ein, bei der von der Klägerin beanspruchten Form eines Damenschuhs handle es sich um einen ganz gewöhnlichen Gebrauchsgegenstand, der keine eigentümliche geistige Schöpfung und daher urheberrechtlich nicht schützbar sei. Der Darstellung eines Damenschuhs aus Schokolade fehle jeder besondere Gedanke und jede originelle Ausgestaltung. Im Geschäftsverkehr würden seit jeher Schuhe in vielen unterschiedlichen Formen aus Schokolade angeboten und vertrieben. Der Schokoladeschuh der Klägerin weiche in keinem einzigen Element vom Alltäglichen, Herkömmlichen ab; er weise keine persönliche, unverwechselbare Note auf und unterscheide sich in keiner Weise von einem gängigen Damenschuh. Auch der Idee, einen Schuh aus Schokolade zu gießen, fehle die Originalität. Die der Beklagten übergebene Gussform der Klägerin sei nach Erfüllung des Auftrags in Vergessenheit geraten und bloß irrtümlich verwendet worden. Unverzüglich nach Geltendmachung der Ansprüche der Klägerin habe die Beklagte die zu Dekorationszwecken in der Auslage ausgestellten Schuhe von dort entfernt, die geforderte Unterlassungserklärung abgegeben, der Klägerin mitgeteilt, dass ein Schuh aus Zucker zu einem Preis von 30 EUR und einer aus Schokolade um 80 EUR verkauft worden sei, und erklärt, 110 EUR an die Klägerin zu überweisen und ihr sämtliche noch bei der Beklagten befindlichen Formen auszufolgen.

Das Erstgericht gab mit Teilurteil dem Unterlassungs-, Beseitigungs-, Rechnungslegungs-, und Veröffentlichungsbegehren statt. Eine urheberrechtlich schützbare (eigentümliche) Schöpfung liege vor, wenn das Werk das Ergebnis schöpferischer Geistestätigkeit sei, das seine Eigenheit, die es von anderen Werken unterscheide, aus der Persönlichkeit seines Schöpfers empfangen habe. Die Persönlichkeit des Urhebers müsse im Werk so zum Ausdruck kommen, dass sie ihm den Stempel der Einmaligkeit und der Zugehörigkeit zu seinem Schöpfer aufpräge. Auf dem Gebiet der bildenden Künste müsse die Gestaltung mit einem gewissen $\mathrm{Maß}$ an Originalität verbunden sein (Werkhöhe). Es müsse eine Gestalt gewordene Idee vorliegen, die den Stempel der persönlichen Eigenart des Schöpfers trage oder sich zumindest durch eine persönliche Note von anderen Erzeugnissen ähnlicher Art abhebe. Die Werkhöhe sei nach der maßgebenden Auffassung der mit künstlerischen Fragen befassten Verkehrskreise zu beurteilen. Da die Klägerin ausgebildete Künstlerin sei und ihr Werk in einer künstlerischen Ausstellung eines weltweit anerkannten Museums ausgestellt worden sei, müsse davon ausgegangen werden, dass das Video-Kunstwerk eine urheberrechtlich geschützte Leistung sei. Dies gelte auch für den Schuh als Teil dieses künstlerischen Werks. Der tragbare Schokoladeschuh der Klägerin genieße somit urheberrechtlichen Schutz, weshalb die Unterlassungs-, Beseitigungs- und Rechnungslegungsansprüche berechtigt seien. Die Urheberrechtsverletzungen der Beklagten seien einem unbestimmten Personenkreis bekannt geworden, dies rechtfertige auch die Urteilsveröffentlichung in einer Tageszeitung.

Das Berufungsgericht hob dieses Urteil auf und verwies die Rechtssache zur neuerlichen Entscheidung an das Erstgericht zurück; es sprach aus, dass der Wert des Entscheidungsgegenstands 20.000 EUR übersteige und der Rekurs an den Obersten Gerichtshof zulässig sei, weil die Lösung der Frage nach dem Werkcharakter von Objekten moderner Kunst in ihrer Bedeutung über den Einzelfall hinausgehe. Der Schokoladeschuh der Klägerin sei Teil eines in einer Ausstellung gezeigten Video-Kunstwerks. Auch getrennt von diesem Umfeld weise das Objekt - besonders "durch die Kombination eines Alltagsgegenstands aus gebrauchsfremdem, sogar essbarem Material mit der Herstellung in (wenngleich wohl nur kurzfristig) tragbarer Schuhgröße" - einen eigenen eigenständigen Reiz und damit individuellen Charakter auf. Durch 
diesen Kontext werde durch die Entscheidung der Schöpferin aus dem Gebrauchsgegenstand ein von anderen Gegenständen abgrenzbares eigenständiges Werk, das urheberrechtlichen Schutz genieße. Es handle sich nicht bloß um ein - gleich den readymades - unverändert ausgewähltes und auf eine neue Bedeutungsebene und damit zur Kunst emporgehobenes Objekt. Die Beklagte sei daher mangels Einräumung von Werknutzungsrechten nicht berechtigt, Werkstücke aus der Gussform der Klägerin herzustellen, zu verkaufen oder in ihrer Auslage auszustellen. Auf den Verwendungszweck der Schuhe komme es insofern ebensowenig an wie auf die Frage, ob es andere Schuhe aus Schokolade in welcher Größe auch immer gebe, habe doch die Beklagte gerade die von der Klägerin entworfene Schuhform verwendet. Die Sache sei aber trotz Bejahung des Werkcharakters wegen einiger Gründe noch nicht spruchreif.

\section{Rechtliche Beurteilung}

Der Rekurs ist zulässig, weil das Berufungsgericht von höchstgerichtlicher Rechtsprechung zum Werkbegriff abgewichen ist; das Rechtsmittel ist berechtigt im Sinn einer Abweisung des Klagebegehrens.

Die Beklagte macht geltend, der Schokoladeschuh der Klägerin sei keine eigentümliche geistige Schöpfung und daher urheberrechtlich nicht schutzfähig; dessen naturgetreue Gestaltung gehe über eine rein handwerkliche Leistung, die sich im Alläglichen und Üblichen bewege, nicht hinaus und entbehre jeglicher individueller Gestaltungselemente.

1.1. Der urheberrechtliche Werkbegriff umschreibt, was den Gegenstand urheberrechtlichen Schutzes ausmacht und im urheberrechtlichen Sinn als "Literatur und Kunst" anzusehen ist. Der urheberrechtliche Kunstbegriff deckt sich nicht notwendig mit dem kunsttheoretischen bzw. einem markt- oder publikumsorientierten, von den jeweiligen Kunstströmungen abhängigen Kunstverständnis, das einem steten Wandel unterliegt. Das urheberrechtlich schützbare Werk muss vielmehr neutral und objektiv umschrieben und für alle künstlerischen Phänomene und Entwicklungen offen sein. Ausgehend von einem offenen Kunstverständnis ist urheberrechtlicher Schutz allen schützenswerten geistigen Schöpfungen zu gewähren, die im weitesten Sinn als Kunst interpretierbar sind und ein Mindestmaß an Gestaltung aufweisen, das sie von anderen (ähnlichen) Produkten unterscheidet. Dieses Mindestmaß an formender Gestaltung wird im Urheberrecht als Originalität, Individualität, Eigenpersönlichkeit oder Eigentümlichkeit beschrieben (Walter Österreichisches Urheberrecht I Rz. 102).

1.2. Urheberechtlich schützbar sind Werke nur, wenn sie eigentümlich im Sinn des § 1 Abs. 1 UrhG sind. Diese Beschränkung folgt im Wesentlichen aus dem urheberrechtlichen Freihaltebedürfnis zu Gunsten der Allgemeinheit. Der urheberrechtliche Schutz jeglicher, auch noch so alltäglicher, banaler Leistungen würde die Schaffensfreiheit anderer unerträglich beeinträchtigen (Walter aaO Rz. 109).
1.3.1. Der Senat geht in ständiger Rechtsprechung davon aus, dass nur eine individuell eigenartige Leistung, die sich vom Alltäglichen, Landläufigen, üblicherweise Hervorgebrachten abhebt, geschützt ist. Die Schöpfung muss zu einem individuellen und originellen Ergebnis geführt haben. Beim Werkschaffenden müssen persönliche Züge - insbesondere durch die visuelle Gestaltung und durch die gedankliche Bearbeitung - zur Geltung kommen. Dem Allerweltserzeugnis, der rein handwerklichen Leistung, die jedermann mit durchschnittlichen Fähigkeiten ebenso zustande bringen würde, fehlt die erforderliche Individualität (Nachweise bei Kucsko in: Kucsko, urheber.recht 89 f.; RIS-Justiz RS0076397, RS0076841 [T12], RS0076435, RS0076367, RS0076913).

1.3.2. Im Zusammenhang mit dem europäischen Werkbegriff hat der Senat bereits wiederholt ausgesprochen, dass es keines besonderen Maßes an Originalität bedarf. Es genügt, dass eine individuelle Zuordnung zwischen Werk und Schöpfer insofern möglich ist, als dessen Persönlichkeit auf Grund der von ihm gewählten Gestaltungsmittel zum Ausdruck kommt und eine Unterscheidbarkeit bewirkt (4 Ob 179/01d = ÖBI 2003, 39 - Eurobike; 4 Ob 274/02a = MR 2003, 162 - Felsritzbild; 4 Ob 103/07m). Diese Rechtsprechung hat im Schrifttum Zustimmung gefunden (Walter aaO Rz. 131 mwN in Fn. 380).

1.4. Da der urheberrechtliche Werkbegriff objektiv konzipiert ist, spielt für die Schutzfähigkeit eines Werks keine Rolle, ob es in Museen ausgestellt, von Publikum und Kunsthandel als Kunst anerkannt, von Kunstsachverständigen als Kunst bewertet oder von einem Künstler geschaffen worden ist (s. auch Walter aaO Rz. 105, 143 m.N. zur Rspr.).

1.5. Der Nachweis des Werkcharakters kann im Allgemeinen schon durch die Vorlage des Werks erbracht werden, weil die Beurteilung, ob dadurch ein Werk iSd UrhG verkörpert wird, eine Rechtsfrage ist, die das Gericht zu lösen hat (4 Ob 19/06g = MR 2006, 264 - Storyboard mwN; vgl RIS-Justiz RS0043530 [T5]).

2.1. Das aus dem Anhang ersichtliche Objekt des Klagebegehrens geht über die naturgetreue Nachformung eines so oder so ähnlich vielfach im Schuhhandel erhältlichen Damenschuhs mit hohem Absatz der Größe 39 1/2 aus purer Schokolade nicht hinaus. Der Schuh weist keinerlei individuelle Gestaltungselemente auf und hebt sich deshalb nicht von einer rein handwerklichen, routinemäßigen Leistung, die sich im Rahmen des Alltäglichen und Üblichen bewegt, ab.

Die Idee, Gegenstände des täglichen Lebens (z.B. Früchte, Autos, Glückssymbole, Münzen, auch Schuhe) naturgetreu oder in verkleinertem Maßstab aus Schokolade oder anderen essbaren Materialien herzustellen und zu verkaufen, hat sich im Süßwarenhandel seit langem durchgesetzt und zu einem nahezu unüberschaubaren Angebot entsprechender Produkte geführt, die einander innerhalb der nachgebildeten Warengattungen ähnlich sind. Da dem von der Klägerin entworfenen Objekt ein Mindestmaß an Eigentümlichkeit und damit formender Gestal- 
tung im zuvor beschriebenen Sinn fehlt, die es von ähnlichen Objekten unterscheidbar machen könnte, fällt es nicht unter den urheberrechtlichen Schutz.

2.2. Dieser Beurteilung lässt sich nicht entgegenhalten, dass der Schokoladeschuh der Klägerin als wesentlicher Bestandteil eines künstlerischen Videofilms entworfen worden sei, der Werkqualität besitze. Wie der Senat erst jüngst erneut ausgeführt hat, ist ein einzelner Werkteil nur unter der Voraussetzung geschützt, dass der betreffende Teil für sich eine eigentümliche geistige Schöpfung iSd § 1 Abs. 1 UrhG ist (4 Ob 111/08i; RISJustiz RS0076935; Kucsko in: Kucsko aaO 100 mwN; Walter aaO Rz. 159). Um den Schutz eines bloßen Werkteils - wie hier - zu beurteilen, ist es somit weder erforderlich, diesen in eine Relation zum Gesamtwerk zu stellen, noch den Werkcharakter des Gesamtwerks zu beurteilen.

2.3. Die Klägerin beruft sich in der Rekursbeantwortung auf die Aussage eines Museumsdirektors, wonach der Schokoladeschuh der Klägerin eine originelle und originäre künstlerische Schöpfung sei, weil er einerseits einen komplexen Ebenentausch vornehme und einen vermeintlichen Gebrauchsgegenstand in ein vermeintlich unmittelbar konsumierbares Schokoladenstück umwandle, andererseits auch den Fetischcharakter, der Damenschuhen mitunter zugeschrieben werde, auf das Beziehungsreichste anschaulich mache.
Diese Beurteilung bewertet den Gegenstand nicht - wie im Urheberrecht notwendig - objektiv für sich allein, sondern setzt ihn in Beziehung zum Videofilm und dem darin zum Ausdruck kommenden Konzept der Klägerin. Objektiv gesehen ist der Schuh allein aber tatsächlich - und nicht nur vermeintlich - ein unmittelbar konsumierbares Schokoladestück, das sich von vergleichbaren Süßigkeiten nicht signifikant unterscheidet.

2.4. Ob das Verhalten der Beklagten unter das lauterkeitsrechtliche Verbot der Produktimitation (§ 2 Abs. 3 Z. 1 UWG) fällt, haben die Vorinstanzen zutreffend nicht geprüft, weil die Klage allein auf urheberrechtliche Ansprüche gestützt worden ist.

3. Der Oberste Gerichtshof kann gemäß § 519 Abs. 2 letzter Satz ZPO über einen Rekurs gegen einen Beschluss des Berufungsgerichts nach $\S 519$ Abs. 1 Z. 2 ZPO durch Urteil in der Sache selbst erkennen, wenn die Sache zur Entscheidung reif ist. Solches ist hier der Fall, weil die auf das Urheberrecht gegründeten Ansprüche nicht berechtigt sind. Der angefochtene Beschluss ist somit aufzuheben und in der Sache selbst im Sinn der Abweisung des Klagebegehrens zu erkennen.

4. Die Kostenentscheidung ist in den $\S \S 41$ Abs. 1, 50 Abs. 1 ZPO begründet. (Entscheidung von der Redaktion bearbeitet) -

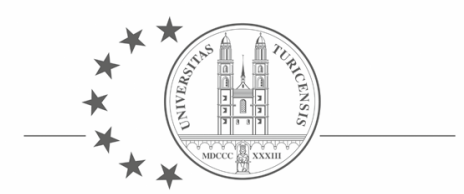 \\ Europa Institut \\ an der Universität Zürich \\ Kompetenzzentrum für Fragen \\ des Europarechts}

Mittwoch, 10. Juni 2009

\section{Kunst und Recht - Nachwehen des Holocaust: 10 Jahre Washingtoner Raubkunst-Richtlinien und Umgang mit Raubkunst in Europa}

Tagungsleitung: Prof. Dr. Kurt Siehr, Dr. Andrea F. G. Raschèr

Museum Rietberg, Gablerstrasse 15, Park-Villa Rieter, 8002 Zürich

Weitere Informationen und Anmeldung: Europa Institut an der Universität Zürich www.eiz.uzh.ch

Tel: +41 / 446344891 Fax: +41 / 446344359 